\title{
Decompressive Craniectomy for Malignant Middle Cerebral Artery Stroke
}

\author{
Mark M. Landreneau, MD ${ }^{1}$ Kevin N. Sheth, MD $^{2}$ \\ 1 Section of Neurocritical Care and Emergency Neurology, Yale \\ University, New Haven, Connecticut \\ 2 Department of Neurology, Yale University, New Haven, Connecticut \\ ${ }^{3}$ Department of Neurosurgery, Yale University, New Haven, \\ Connecticut
}

Semin Respir Crit Care Med 2017;38:737-744.

\begin{abstract}
Address for correspondence Kevin N. Sheth, MD, Department of Neurology, Yale University, PO Box 208018, New Haven, CT 06520 (e-mail: kevin.sheth@yale.edu).
\end{abstract}
Abstract
Keywords
- Decompressive Craniectomy
- decompressive hemicraniectomy
- malignant
- ischemic stroke
- middle cerebral artery stroke
- hemispheric stroke
- malignant edema
- surgical decompression
- stroke edema

Advancements in the treatment of ischemic stroke have led to a recent decline in overall stroke mortality, but patients with hemispheric infarcts remain at high risk for death. Recent advances in the approach to this devastating disease include early identification of patients at high risk for swelling and standardized approaches to medical therapy. However, surgical decompression continues to be the most effective treatment for malignant edema from large hemispheric strokes. Patient selection in the past had been strictly limited to younger ages and the nondominant hemisphere. More recent evidence demonstrates a mortality benefit in older patients with a limited impact on morbidity. Judicious patient selection and shared, informed decision making with families remain the optimal approach for this devastating disease.
Large ischemic infarcts of the middle cerebral artery (MCA) territory carry significant risks of morbidity and mortality due to not only the destruction of a large volume of brain but also the progressive and deleterious effects of poststroke edema. ${ }^{1}$ Edema associated with large strokes follows a typical crescendo-decrescendo pattern over several days, in some cases leading to transtentorial herniation and progressive neurological decline, making them "malignant" (-Fig. 1). About $10 \%$ of supratentorial infarcts fall into this classification. In large prospective studies of these patients, mortality rates approach $60 \%^{2}$ for patients treated with medical therapy alone, though there likely exist some bias due to the self-fulfilling prophecy of early withdrawal of life sustaining therapies. Many surviving patients are left with profound neurological disability. Approximately two-thirds of survivors remain completely dependent on others (modified Rankin scale [mRS] 4-5). ${ }^{2}$

In light of these dispiriting facts, there remains some degree of controversy over the best approach to patients with malignant MCA stroke syndromes. The cumulative evidence over the past several years comparing standard of care medical therapy to surgical decompressive craniectomy has shown survival benefit and a trend to improved outcomes, and it remains the recommendation of the American Heart Association (AHA)/American Stroke Association (ASA) ${ }^{3}$ and Neurocritical Care Society, ${ }^{4}$ particularly for younger patients. As the current therapy exists, however, intervention often introduces a tradeoff between mortality and severe disability, which requires an individualized approach to care and patient values.
Issue Theme Advancements in Neurocritical Care and Emergency Neurology; Guest Editors: David Y. Hwang, MD, FNCS, and David M. Greer, MD, MA, FCCM, FAHA, FNCS, FAAN, FANA
DOI https://doi.org/ $10.1055 / \mathrm{s}-0037-1607992$. ISSN 1069-3424. 

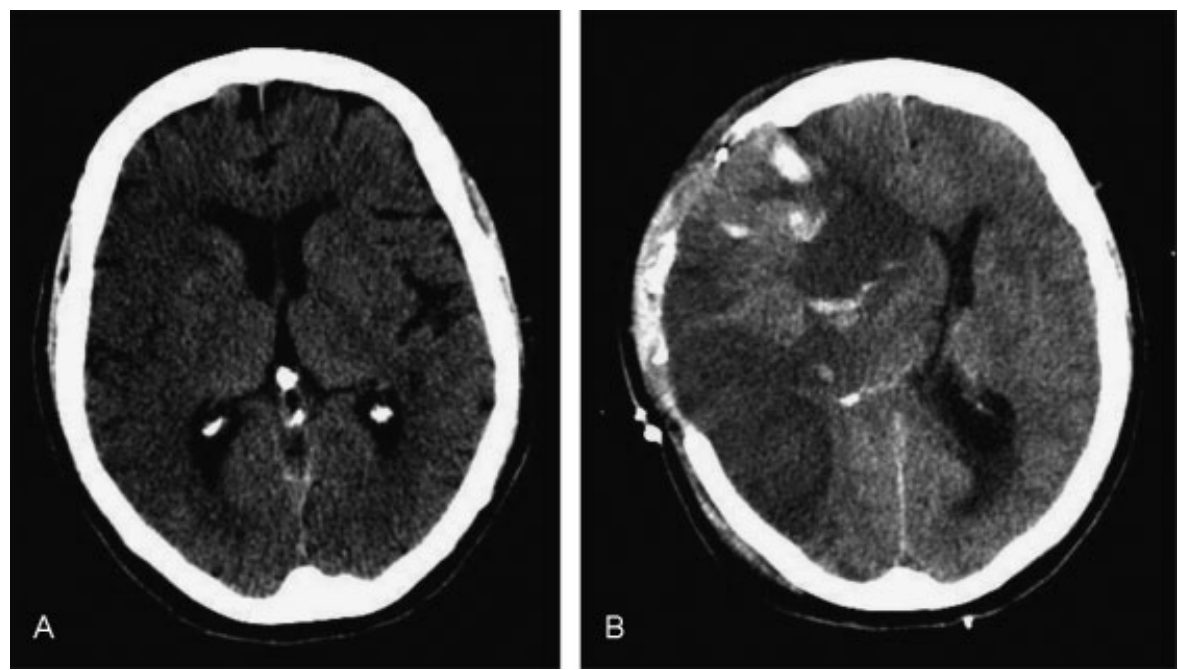

Fig. 1 Noncontrast head CT of a patient with a malignant right middle cerebral artery infarct (A) before and (B) after decompressive hemicraniectomy. Preoperative CT shows large territory of hypodensity. Postoperative scan demonstrates edema, hemorrhagic transformation, and mass effect. CT, computed tomography.

\section{Predictors of Malignant Edema and Herniation}

\section{Clinical Features}

In the anterior circulation, large hemispheric infarcts are most commonly embolic or thrombotic occlusions of the terminus of the internal carotid artery, "carotid T," or of the proximal segment of the MCA, radiographically designated as M1. ${ }^{1}$ The clinical syndrome is typically sudden onset of profound deficits-hemiplegia, gaze deviation, homonymous quadrantanopsia or hemianopsia, and either aphasia or neglect, depending on hemisphere. As the infarcted tissue swells, the syndrome can progress to decreased levels of consciousness because of elevated intracranial pressure (ICP) or distortion of bilateral projections from the reticular activating system. ${ }^{5}$ of note, a large randomized prospective study has shown the early appearance of decreased arousal is a poor prognostic sign. ${ }^{6}$ Delayed focal findings such as pupillary dilatation, cranial nerve palsies, and ipsilateral hemiparesis can appear due to herniation of the temporal lobe against the midbrain.

In large retrospective case series, there have been several clinical features identified as independent predictors of developing a malignant MCA syndrome. A major epidemiological risk factor is younger age, 7,8 which is in part due to a combination of larger brain volume compared with older patients with decreased intracranial space for swelling. ${ }^{1,7} \mathrm{~A}$ postmortem analysis ${ }^{9}$ also found specific features of patients who died of malignant infarcts including no history of stroke, female sex, elevated heart weight, abnormal ipsilateral circle of Willis, and carotid occlusion. Other independent risk factors shown to be predictors of malignant infarction include a history of hypertension, which may lead to a poor autoregulation and impaired collateral perfusion ${ }^{10,11}$; systolic blood pressure $>180 \mathrm{~mm} \mathrm{Hg}$ after 12 hours from onset $^{7}$; and a history of heart failure as well as elevated peripheral white blood cell count. ${ }^{10}$

\section{Radiographic Features}

\section{Computed Tomography}

Advanced imaging such as diffusion magnetic resonance imaging (MRI), perfusion computed tomography (CT), and MRI are becoming more widely used in the assessment of acute stroke, but noncontrast head CT remains the most widely used neuroimaging study in acute stroke. Two large retrospective case-control studies ${ }^{7,10}$ demonstrated that involvement of more than $50 \%$ of the MCA territory on head CT at 2 to 3 hours poststroke onset was an independent predictor of fatal brain edema. When studied prospectively, ${ }^{12}$ more than $80 \%$ of patients with $>50 \%$ infarction on CT within first 3 hours developed a fatal malignant MCA syndrome. In the same study, additional findings with high positive predictive value (PPV) for death from malignant edema included local brain swelling (70\% PPV) and a hyperdense MCA trunk (32\% PPV). Quantified, an infarct volume $>220 \mathrm{~mL}$ and midline shift $>3.9 \mathrm{~mm}$ are predictive of severe brain edema leading to herniation. ${ }^{13}$ Alberta Stroke Program Early CT Score (ASPECTS) can be used as a more formal grading system, and scores $<7$ are associated with progression to malignant infarction (50\% sensitivity and $85 \%$ specificity). ${ }^{14}$

\section{Computed Tomography Perfusion and Flow Studies}

The major limitation of noncontrast head CT is poor sensitivity, particularly early in the clinical course of stroke, a limitation that can be improved with the addition of contrast-based perfusion studies to identify large core infarcts. An infarct core volume greater than two-thirds of the MCA territory on CT perfusion predicted malignant edema with high sensitivity (92\%) and high specificity (94\%). ${ }^{15}$ When additional large vessel territories are involved as core infarct, particularly with carotid occlusions, the likelihood of malignant infarct is even higher. ${ }^{16}$ Furthermore, in large strokes (National Institutes of Health Stroke Scale [NIHSS] >18), poor 
collateral circulation scores were independently associated with malignant infarction. ${ }^{17}$

Xenon CT scanning can also been used for early identification of malignant stroke syndromes. In a retrospective study of 20 patients, ${ }^{18}$ a xenon CT scan within 6 hours of symptom onset that demonstrated hemispheric cerebral blood flow $\leq 15 \mathrm{~mL} / 100 \mathrm{~g}$ per minute was associated with severe edema and herniation (sensitivity 100\% and specificity $50 \%)$.

\section{Magnetic Resonance Imaging}

Compared with noncontrast head CT, MRI of the brain better predicts malignant edema in the earliest stages of infarction. In particular, diffusion-weighted imaging (DWI) has shown to have high sensitivity and specificity for predicting malignant edema. In one series of 28 patients, ${ }^{19}$ DWI infarct volumes $>145 \mathrm{~mL}$ within 14 hours of symptom onset were predictive of malignant infarction (100\% sensitivity and $94 \%$ specificity). A larger prospective study of 140 patients ${ }^{20}$ showed that DWI volumes within 6 hours of symptom onset $>82 \mathrm{~mL}$ predicted malignant infarction with high specificity (98\%) but low sensitivity (52\%). A retrospective analysis ${ }^{21}$ showed that using MRI to measure the degree of brain atrophy can further increase PPV of lower volume DWI lesions $(>87 \mathrm{~mL}$ ) for the development of malignant edema (0.93 vs. 0.70$)$.

\section{Tissue Metabolism Imaging}

Small studies of single photon emission CT (SPECT) and 11C flumazenil positron emission tomography (PET) have shown they can be used to assess volumes of infarct core and predict malignant course with a high sensitivity. When performed in the first 24 hours of symptom onset, the sensitivity of SPECT in predicting herniation due to brain edema has been shown to have higher sensitivity than noncontrast head CT ( 82 vs. $36 \%$ ) with similar specificity. ${ }^{22}$ Likewise, in a study of 34 patients with early CT changes showing ischemic changes in $>50 \%$ territory of MCA territory, flumazenil PET was shown to be useful to predict malignant course. ${ }^{23}$ Patients with malignant courses had a larger mean ischemic core than those with benign courses, 144.5 versus $62.2 \mathrm{~mL}$.

\section{Biomarkers and Serum Markers}

Though biomarkers have some utility as corroborative data in conjunction with imaging and clinical data, they have not shown readily available utility as independent predictors. Invasive monitoring with microdialysis of cerebral spinal fluid in the area of the stroke bed has demonstrated elevations in excitatory neurotransmitters such as glutamate and aspartate in addition to an anaerobic metabolic profile with in lactate and lactate/pyruvate ratios in patients who went on the develop malignant syndromes. ${ }^{23}$

There are a few serum markers that have been studied as potentially useful for predicting malignant course. A serum protein marker of glial injury, SB100, has been shown to rise in proportion with size of infarct. ${ }^{24}$ Significant elevations in the first 12 to 24 hours poststroke have been associated with an increased risk of herniation in patients with large MCA strokes. ${ }^{25}$ Another small case-control study showed serum cellular fibronectin could predict a malignant course when present in very elevated levels $(>16.6 \mu \mathrm{g} / \mathrm{mL}) .{ }^{26}$ There is also some evidence that high matrix metalloproteinase 9 levels are associated with increased vasogenic edema and risk of malignant infarct. ${ }^{27}$

\section{Predictive Models}

Multiple clinical grading scales have been developed to better predict risk of a developing a malignant MCA syndrome. The EDEMA ${ }^{28}$ score is a proposed score that uses basal cistern effacement (yes: 3 ; no: 0 ), serum glucose ( $\geq 150: 2 ;<150: 0)$, midline shift (>9 mm: 7; 6-9 mm: 4; 3-6 mm: 2; 0-3 mm: 1; $0 \mathrm{~mm}: 0$ ), previous stroke (no: 1 ; yes: 0 ), and tissue plasminogen activator or thrombectomy (no: 1 ; yes: 0 ) as predictive variables for malignant edema. It has not yet been validated, but a score $>7$ confers a 93\% PPV for development of malignant edema.

The DASH ${ }^{29}$ score is another predictive model that uses four variables: DWI ASPECTS ( $\leq 3$ : 1 point; $>3$ : 0 point), anterior cerebral artery territory involvement (yes: 1 point; no: 0 point), M1 susceptibility vessel sign (yes: 1 point; no 0: point), and hyperglycemia ( $\geq 145$ : 1 point; $<145$ : 0 point). The likelihood of developing a malignant infarction was scaled according to score. A score of 0 was $9.1 \%$; score 1 : 20.5\%; score 2: $63.0 \%$; and scores 3 and 4: $96.8 \%$.

Another score ${ }^{30}$ has used NIHSS ( $\geq 18$ : 2 points; 9-17: 1 point; $\leq 9$ : 0 point) ASPECTS ( $\leq 7: 1$ point; $>8$ : 0 point), collateral score ( $<2: 2$ points; $\geq 2$ : 0 point), and revascularization failure (yes: 1 point; no: 0 point). More than $80 \%$ of patients with a score of 5 and all patients with a score of 6 developed malignant brain edema.

\section{Treatment}

\section{Decompressive Craniectomy}

Traditional medical management of malignant MCA stroke involves the use of hyperosmolar therapy, sedation, and hyperventilation. The evidence for significant mortality and morbidity benefit for these conservative therapies remains unclear. ${ }^{31-34}$ Intravenous glyburide has shown some evidence of reducing stroke-associated edema ${ }^{35}$ but remains in early stages of investigation.

Because of the current limitations of medical therapy in controlling malignant edema, decompressive craniectomy provides an adjunctive measure to allow for brain expansion outside the cranial vault. The surgery involves incising and reflecting a large portion of the scalp to expose the frontal, parietal, and temporal bones. A large bone flap with wide margins around the stroke bed is then removed. Current recommendations are for a bone flap of at least $12 \mathrm{~cm},{ }^{4}$ and possibly up to 13 to $14 \mathrm{~cm}$ for some patients. ${ }^{36}$ The bone flap is then stored in a refrigerated tissue bank or intraperitoneally. A cruciate incised durotomy is performed to allow for swelling out of the cranial vault, and in some cases, necrotic tissue may be removed, although this is typically avoided to preserve intermixed areas of healthy tissue within the stroke bed. The goal of therapy is to reduce ICP, improve regional 
perfusion, decrease midline shift, and prevent fatal compression of the brain stem due to edematous brain.

One of the first reports of using decompressive craniectomy for stroke was a case series in $1956 .{ }^{37}$ Once CT and advanced imaging became more widespread, early detection of stroke led to more frequent consideration of decompression. Several case series ${ }^{38}$ demonstrated a trend toward survival benefit with decompressive craniectomy, which prompted investigation with multiple prospective randomized controlled trials. Overall, these trials have demonstrated a mortality benefit with early decompression in addition to aggressive medical care, but survivors are often left with moderate-to-severe disability. Questions of the net benefit of decompression remain in light of this mortality disability trade-off, ${ }^{39}$ but careful patient selection can provide a subset of patients not just with a significant mortality but also morbidity benefit.

\section{Outcomes Data}

Two large meta-analyses have been performed on currently available clinical trial data. The first review in $2015^{40}$ evaluated six high-quality trials from 2007 to 2014: DECIMAL, ${ }^{41}$ DESTINY ${ }^{42}$ HAMLET, ${ }^{43}$ DESTINY II, ${ }^{44}$ HeADDFIRST, ${ }^{45}$ and another large prospective trial. ${ }^{46} \mathrm{~A}$ total of 317 patients were included in the analysis. Criteria for inclusion were patients with a malignant MCA stroke who were randomized to medical therapy alone or medical therapy and surgical decompression. Follow-up was performed at 6 and 12 months using the mRS. Several conclusions were drawn from the following pooled analysis:

1. There is a significant survival benefit to surgical decompression. The odds ratio for death (mRS 6) in the decompressive surgery group compared with standard medical management group was statistically significant at 0.17 (95\% CI: 0.10-0.29).

2. The frequency of patients with severe disability in the surgical decompression group was higher compared with medical therapy. The number of patients with a mRS of 3 or 5 was higher in the decompressive surgery group but did not reach statistical significance. The number of patients with a mRS of 4 was statistically significant; odds ratio [OR] of 4.43 (95\% CI: 2.27-8.66).

3. Some patients had a significant morbidity benefit. The number of patients with mRS of 2 was significantly higher in the surgical decompression group with an OR of 4.51 (95\% CI: 1.06-19.24).

4. Previous reviews ${ }^{38}$ suggested benefit only up to the age of 60 years for surgical decompression, but the researchers here found mortality benefit extends up to the age of 80 years. Three trials in the dataset included a large number of older patients: DESTINY $\mathrm{II}^{44}$ included only patients of 61 to 80 years old, Frank et $\mathrm{al}^{45}$ included patients up to the age of 75 years, and Zhao et $\mathrm{al}^{46}$ included patients up to the age of 80 years.

Another large meta-analysis in $2016^{2}$ had many similar conclusions to the one the year prior. A total of 338 patients were included from seven trials: DECIMAL, ${ }^{47}$ DESTINY, ${ }^{42}$ DESTINY II, ${ }^{44}$ HAMLET, ${ }^{43}$ HeADDFIRST, ${ }^{45}$ Zhao et al, ${ }^{46}$ and
Slezins et al. ${ }^{48}$ They made the following observations based on 12-month follow-up data:

1. There was a significant reduction in mortality in the surgical decompression group compared with best medical management. The craniectomy group had 39\% fewer deaths. The chance of being a survivor in the surgical group had a relative risk (RR) of 2.05 (95\% CI: 1.54-2.72). The quality of evidence for this finding was high.

2. Severe disability was higher in the surgical decompression group. There were $4 \%$ more patients in the mRS 5 group and $22 \%$ more in mRS 4 . The quality of evidence for this finding was moderate.

3. Mild-to-moderate disability was also increased in the surgical group. The chances of surviving with a mRS of 3 or less by RR of 1.58 (95\% CI: 1.02-2.46). However, the quality of evidence for this finding was low.

4. The impact of age older than 60 years was not significant with respect to mortality risk.

5. Timing of surgery within 48 or 96 hours did not have a significant impact on outcome.

\section{Further Considerations}

Given the evidence that shows a significant mortality benefit to decompressive craniectomy as well as a significant trend toward reduced morbidity, the remaining considerations include age, decompression of dominant versus nondominant hemisphere stroke, timing of surgery, procedural complications, cost, and most importantly considerations of patient values.

Age

The DESTINY II $^{44}$ trial provided the first and only dedicated randomized controlled data for surgical decompression versus maximal medical therapy in older patients with malignant MCA infarct. The study showed that for patients, age 60 to 80 years, there was a significant mortality benefit of surgical decompression. At 1 year, mortality was $76 \%$ in the medical control arm versus $43 \%$ in the surgical decompression arm.

However, $51 \%$ of patients in the surgical arm had a $m R S \geq 4$ compared with $19 \%$ in the medical arm. Only $5 \%$ of patients in the surgical arm had a mRS of 3 , there were none with a mRS of $<3$. This number is substantially lower compared with the combined results of previous randomized trials in all patients, where $>25 \%$ of patients in the surgical arm had a mRS of $\leq 3 .{ }^{2,40}$ These results show there is a significant risk that decompressive craniectomy will be a lifesaving but debilitating procedure, and though it is not an exclusionary criteria, the strong need for informed decision making with older patients and their decision makers is clear (-Fig. 2).

\section{Dominant versus Nondominant Hemisphere}

Because of the high risk of residual severe aphasia in patients with dominant hemisphere malignant syndromes, offering lifesaving craniectomy remains a somewhat controversial choice. Functional outcome scores such as the mRS do not adequately capture differences between hemiplegic patients with respect to language as both will score 4 or 5 . However, 


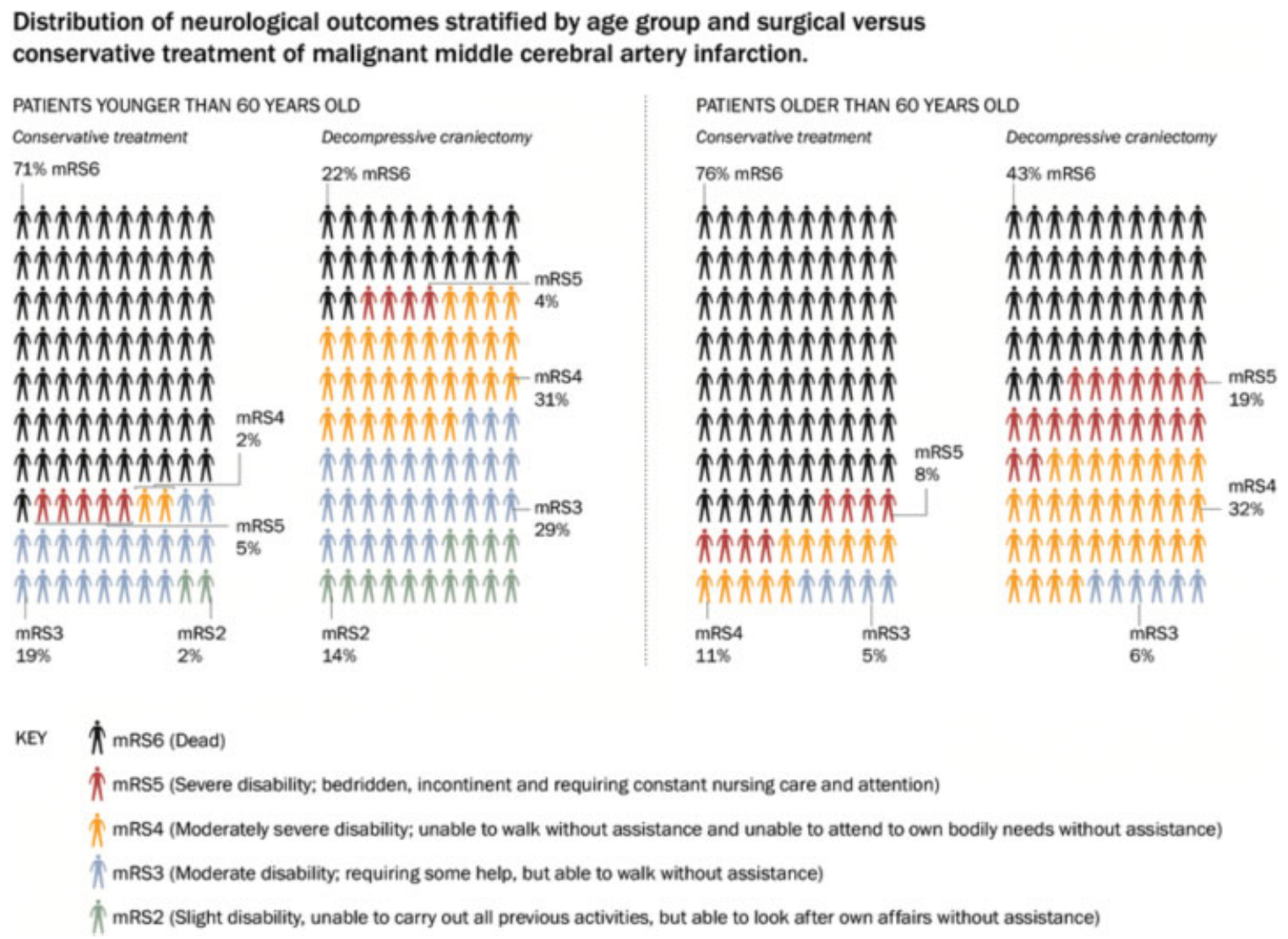

Fig. 2 Representation of neurological outcomes between medical and surgical treatment arms. For patients younger than 60 years, data pooled from DESTINY I, HAMLET, and DECIMAL. For patients older than 60 years, data are represented from DESTINY II. mRS, modified Rankin scale.

this consideration should be tempered by the recognition that nondominant hemisphere strokes can be profoundly disabling apart from motor function due to neglect syndromes as well as more subtle cognitive, emotional, and behavioral changes. Furthermore, there is limited data showing significant improvement in aphasia postcraniectomy for malignant stroke. One study followed a group of 14 patients, and in 13, a significant language improvement was seen at 1 year. ${ }^{49}$ Furthermore, patients preferences may not be as straightforward as expected, and as one study ${ }^{50}$ has shown that global aphasia may be considered better than hemiplegia, which was for many to be considered worse than death. Though the likelihood of significant disability with or without aphasia should be communicated to decision makers, no strict exclusionary principle should be applied to patients with dominant hemisphere stroke in light of these complicated and individual factors.

\section{Timing of Surgery}

As a theoretical point, it stands that early surgery, prior to the signs of herniation, would offer best clinical outcomes for decompressive craniectomy. Avoiding significant elevations in ICP and clinically apparent signs of herniation would seem to offer the best chance at preserving maximal brain tissue. Multiple animal models ${ }^{51,52}$ have suggested as much, but clinical evidence for significant benefit from early surgery has not been as robust.
Nonrandomized data on patient outcomes has not had any definitive suggestion of benefit for early treatment. A 2004 review of nonrandomized studies of patients treated with craniectomy ${ }^{38}$ did not show a significant difference in outcomes based on time to treatment. Another retrospective study of 1,301 patients who had decompressive craniectomy for stroke showed a mild increased risk of disability, in particular, discharge to a facility associated with surgery after 48 hours (OR: 1.17, 95\% CI: 1.05-1.31), but these data were not controlled for confounders such as age. ${ }^{53}$

The data from randomized controlled trials are, in short, insufficient to draw a conclusion regarding the benefits of earlier surgery. Based on enrollment patterns of the large randomized controlled trials for decompression, patients can be grouped into very early surgery $(<24$ hours postonset of symptoms), early surgery (24-48 hours), and delayed (48-96 hours). A pooled analysis of three early randomized controlled trials ${ }^{47}$ showed no difference between very early or early surgery with respect of mortality or functional outcome. In the HAMLET ${ }^{54}$ and HeADDFIRST ${ }^{45}$ trials, some patients enrolled in the surgical arm received decompressive craniectomy up to 96 hours postsymptom onset. In HAMLET trial, 11 patients received surgery after 48 hours, and HeADDFIRST trial had only 8 patients with late decompression. Compared with early surgery, there was no statistically significant difference in mortality, and the numbers were too small to draw any definitive conclusions about benefits, 
though there was a trend toward a benefit in functional outcome with early surgery. As there was no randomization regarding timing of treatment, patient selection bias cannot be excluded and until a dedicated randomized controlled trial examining early versus late surgery is performed, there is no evidence-based strict exclusion to delayed surgery.

\section{Procedural and Postprocedural Complications}

There are several acute and chronic complications of decompressive craniectomy. ${ }^{55}$ Acute complications include hydrocephalus, infection, seizures, and external brain tamponade. Chronic complications include sinking skin flap syndrome and paradoxical herniation. A study of the DECIMAL trial ${ }^{56}$ showed $\sim 10 \%$ of patients developed symptomatic sinking skin flap syndrome, and another $15 \%$ had asymptomatic radiographic evidence of SSFS.

Sinking skin flap syndrome is a common chronic complication of craniectomy due to atmospheric pressure causing large brain tissue shifts due to low ICP. The area beneath a skull defect will appear depressed and shifted toward the contralateral side. If left untreated, it can become symptomatic, causing a low pressure headache or focal weakness, and in the most extreme cases, it can progress to paradoxical brain herniation, coma, and death. Definitive treatment is cranioplasty and bridging therapies include clamping cerebrospinal fluid draining, Trendelenburg position, volume resuscitation, avoidance of hyperosmolar therapy and hyperventilation, and head positioning with skin flap site down. ${ }^{56}$

\section{Patient Values and Quality of Life}

Only two of the major randomized controlled trials for decompressive craniectomy used quality-of-life measurements in their outcomes assessment. In HAMLET trial, ${ }^{54}$ quality of life was measured by Medical Outcome Study $(\mathrm{SF}-86)^{57}$ and a visual analog scale. ${ }^{58}$ They did not find any significant differences between the medical and surgical groups except for the physical summary score, which was better in the medical arm.

The DECIMAL trial ${ }^{41}$ also evaluated quality-of-life outcomes via the French version of the stroke impact scale, ${ }^{59}$ an eight-domain scale with four psychosocial and four physical domains. There were no significant differences in quality of life between the groups, and the authors noted that all survivors were able to acknowledge "life is worth living."

Further reports rely on limited data from trials and observational studies. One study found a majority of patients who survive would have chosen the same course if they had the option again. ${ }^{60}$ Another found that among 64 survivors of craniectomy who had undergone rehabilitation and recovery, $69 \%$ of patients and families would consider decompressive craniectomy again if they had to make the choice again. ${ }^{38}$ With that said, the HAMLET study found that more than $70 \%$ of caregivers experienced high levels of stress in their daily lives in 3 years after stroke. ${ }^{43}$

\section{Cost}

High rates of disability among survivors of craniectomy have introduced concerns not only the quality of life of survivors but also the costs. Investigators of the HAMLET ${ }^{54}$ study performed a cost-effectiveness analysis of surgical decompression for the first 3 years comparing patients treated in the surgical decompression arm versus the medical treatment arm. ${ }^{61}$ They found that compared with medical therapy, surgical craniectomy increases quality-adjusted life years (QALYs) at a mean difference of 1.0 QALY (95\% CI: $0.6-1.4)$ but a high cost, a mean difference of $€ 127,000^{\mathrm{a}}(95 \%$ CI: 73,100-181,000) per QALY gained in the first 3 years. Estimated over a lifetime, they predicted approximately $€ 60,000$ per QALY gained.

\section{Recommendations}

A Statement for Healthcare Professionals from the Neurocritical Care Society and the German Society of Neuro-Intensive Care and Emergency Medicine ${ }^{4}$ has the following evidencebased recommendations ${ }^{3}$ :

1. Decompressive craniectomy after hemispheric infarct (strong recommendations, high quality of evidence)

2. For patients $>60$ years of age, a greater reliance on patient and family input (strong recommendation, moderate quality of evidence)

3. Early decompression (24-48 hours after onset) prior to herniation symptoms (strong recommendation, moderate quality of evidence).

These recommendations are in line with previous guidelines of the AHA/ASA ${ }^{3}$ but updated with evidence on older patients from the interim DESTINY II $^{61}$ trial.

In light of the available evidence and therapeutic options, we recommend that for patients younger than 60 years with an infarct of at least $50 \%$ of the MCA territory and high risk for progression to a malignant syndrome, aggressive medical management with early decompressive craniectomy ( $<48$ hours) prior to the onset of signs of brain stem injury. Decompression may also be considered for patients within a 96-hour window. For patients who are older than 60 years, surgical decompression may remain an option but pursued after thorough discussion with primary decision makers regarding patient values given the high rates of severe disability in survivors.

\section{References}

1 Hacke W, Schwab S, Horn M, Spranger M, De Georgia M, von Kummer R. 'Malignant' middle cerebral artery territory infarction: clinical course and prognostic signs. Arch Neurol 1996;53 (04):309-315

2 Alexander P, Heels-Ansdell D, Siemieniuk R, et al. Hemicraniectomy versus medical treatment with large MCA infarct: a review and meta-analysis. BMJ Open 2016;6(11):e014390

3 Wijdicks EF, Sheth KN, Carter BS, et al; American Heart Association Stroke Council. Recommendations for the management of cerebral and cerebellar infarction with swelling: a statement for healthcare professionals from the American Heart Association/ American Stroke Association. Stroke 2014;45(04):1222-1238

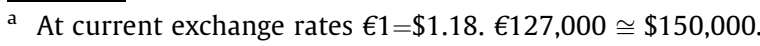


4 Torbey MT, Bösel J, Rhoney DH, et al. Evidence-based guidelines for the management of large hemispheric infarction : a statement for health care professionals from the Neurocritical Care Society and the German Society for Neuro-intensive Care and Emergency Medicine. Neurocrit Care 2015;22(01):146-164

5 Ropper AH. Lateral displacement of the brain and level of consciousness in patients with an acute hemispheral mass. $\mathrm{N}$ Engl J Med 1986;314(15):953-958

6 Cucchiara BL, Kasner SE, Wolk DA, et al; CLASS-I Investigators. Early impairment in consciousness predicts mortality after hemispheric ischemic stroke. Crit Care Med 2004;32(01):241-245

7 Krieger DW, Demchuk AM, Kasner SE, Jauss M, Hantson L. Early clinical and radiological predictors of fatal brain swelling in ischemic stroke. Stroke 1999;30(02):287-292

$8 \mathrm{Ng}$ LK, Nimmannitya J. Massive cerebral infarction with severe brain swelling: a clinicopathological study. Stroke 1970;1(03): 158-163

9 Jaramillo A, Góngora-Rivera F, Labreuche J, Hauw JJ, Amarenco P. Predictors for malignant middle cerebral artery infarctions: a postmortem analysis. Neurology 2006;66(06):815-820

10 Kasner SE, Demchuk AM, Berrouschot J, et al. Predictors of fatal brain edema in massive hemispheric ischemic stroke. Stroke 2001;32(09):2117-2123

11 Williams JL, Furlan AJ. Cerebral vascular physiology in hypertensive disease. Neurosurg Clin N Am 1992;3(03):509-520

12 von Kummer R, Meyding-Lamadé U, Forsting M, et al. Sensitivity and prognostic value of early $\mathrm{CT}$ in occlusion of the middle cerebral artery trunk. AJNR Am J Neuroradiol 1994;15(01): 9-15, discussion 16-18

13 Park J, Goh DH, Sung JK, Hwang YH, Kang DH, Kim Y. Timely assessment of infarct volume and brain atrophy in acute hemispheric infarction for early surgical decompression: strict cutoff criteria with high specificity. Acta Neurochir (Wien) 2012;154 (01):79-85

14 MacCallum C, Churilov L, Mitchell P, Dowling R, Yan B. Low Alberta Stroke Program Early CT score (ASPECTS) associated with malignant middle cerebral artery infarction. Cerebrovasc Dis 2014;38 (01):39-45

15 Ryoo JW, Na DG, Kim SS, et al. Malignant middle cerebral artery infarction in hyperacute ischemic stroke: evaluation with multiphasic perfusion computed tomography maps. J Comput Assist Tomogr 2004;28(01):55-62

16 Kucinski T, Koch C, Grzyska U, Freitag HJ, Krömer H, Zeumer H. The predictive value of early $\mathrm{CT}$ and angiography for fatal hemispheric swelling in acute stroke. AJNR Am J Neuroradiol 1998;19(05): 839-846

17 Kim H, Jin ST, Kim YW, Kim SR, Park IS, Jo KW. Predictors of malignant brain edema in middle cerebral artery infarction observed on CT angiography. J Clin Neurosci 2015;22(03): 554-560

18 Firlik AD, Yonas H, Kaufmann AM, et al. Relationship between cerebral blood flow and the development of swelling and lifethreatening herniation in acute ischemic stroke. J Neurosurg 1998;89(02):243-249

19 Oppenheim C, Samson Y, Manaï R, et al. Prediction of malignant middle cerebral artery infarction by diffusion-weighted imaging. Stroke 2000;31(09):2175-2181

20 Thomalla G, Hartmann F, Juettler E, et al; Clinical Trial Net of the German Competence Network Stroke. Prediction of malignant middle cerebral artery infarction by magnetic resonance imaging within 6 hours of symptom onset: A prospective multicenter observational study. Ann Neurol 2010;68(04):435-445

21 Beck C, Kruetzelmann A, Forkert ND, et al. A simple brain atrophy measure improves the prediction of malignant middle cerebral artery infarction by acute DWI lesion volume. J Neurol 2014;261 (06):1097-1103

22 Berrouschot J, Barthel H, von Kummer R, Knapp WH, Hesse S, Schneider D. $99 \mathrm{~m}$ technetium-ethyl-cysteinate-dimer single- photon emission CT can predict fatal ischemic brain edema. Stroke 1998;29(12):2556-2562

23 Dohmen C, Bosche B, Graf R, et al. Prediction of malignant course in MCA infarction by PET and microdialysis. Stroke 2003;34(09): 2152-2158

24 Büttner T, Weyers S, Postert T, Sprengelmeyer R, Kuhn W. S-100 protein: serum marker of focal brain damage after ischemic territorial MCA infarction. Stroke 1997;28(10):1961-1965

25 Foerch C, Otto B, Singer OC, et al. Serum S100B predicts a malignant course of infarction in patients with acute middle cerebral artery occlusion. Stroke 2004;35(09):2160-2164

26 Serena J, Blanco M, Castellanos M, et al. The prediction of malignant cerebral infarction by molecular brain barrier disruption markers. Stroke 2005;36(09):1921-1926

27 Zhao BQ Tejima E, Lo EH. Neurovascular proteases in brain injury, hemorrhage and remodeling after stroke. Stroke 2007;38(2, Suppl):748-752

28 Ong CJ, Gluckstein J, Laurido-Soto O, Yan Y, Dhar R, Lee JM. Enhanced detection of Edema in Malignant Anterior Circulation Stroke (EDEMA) score: a risk prediction tool. Stroke 2017;48(07): 1969-1972

29 Shimoyama T, Kimura K, Uemura J, et al. The DASH score: a simple score to assess risk for development of malignant middle cerebral artery infarction. J Neurol Sci 2014;338(1-2):102-106

30 Jo K, Bajgur SS, Kim H, Choi HA, Huh PW, Lee K. A simple prediction score system for malignant brain edema progression in large hemispheric infarction. PLoS One 2017;12(02):e0171425

31 Bereczki D, Fekete I, Prado GF, Liu M. Mannitol for acute stroke. Cochrane Database Syst Rev 2007;(03):CD001153

32 Righetti E, Celani MG, Cantisani T, Sterzi R, Boysen G, Ricci S. Glycerol for acute stroke. Cochrane Database Syst Rev 2004;(02): CD000096

33 Hofmeijer J, van der Worp HB, Kappelle LJ. Treatment of spaceoccupying cerebral infarction. Crit Care Med 2003;31(02):617-625

34 Sandercock PA, Soane T. Corticosteroids for acute ischaemic stroke. Cochrane Database Syst Rev 2011;(09):CD000064

35 Kimberly WT, Battey TW, Pham L, et al. Glyburide is associated with attenuated vasogenic edema in stroke patients. Neurocrit Care 2014;20(02):193-201

36 Curry WT Jr, Sethi MK, Ogilvy CS, Carter BS. Factors associated with outcome after hemicraniectomy for large middle cerebral artery territory infarction. Neurosurgery 2005;56(04):681-692, discussion 681-692

37 Scarcella G. Encephalomalacia simulating the clinical and radiological aspects of brain tumor; a report of 6 cases. J Neurosurg 1956;13(04):278-292

38 Gupta R, Connolly ES, Mayer S, Elkind MS. Hemicraniectomy for massive middle cerebral artery territory infarction: a systematic review. Stroke 2004;35(02):539-543

39 Honeybul S, Ho KM, Gillett G. Outcome following decompressive hemicraniectomy for malignant cerebral infarction: ethical considerations. Stroke 2015;46(09):2695-2698

40 Back L, Nagaraja V, Kapur A, Eslick GD. Role of decompressive hemicraniectomy in extensive middle cerebral artery strokes: a meta-analysis of randomised trials. Intern Med J 2015;45(07): 711-717

41 Vahedi K, Vicaut E, Mateo J, et al; DECIMAL Investigators. Sequential-design, multicenter, randomized, controlled trial of early decompressive craniectomy in malignant middle cerebral artery infarction (DECIMAL trial). Stroke 2007;38(09):2506-2517

42 Jüttler E, Schwab S, Schmiedek P, et al; DESTINY Study Group. Decompressive Surgery for the Treatment of Malignant Infarction of the Middle Cerebral Artery (DESTINY): a randomized, controlled trial. Stroke 2007;38(09):2518-2525

43 Geurts M, van der Worp HB, Kappelle LJ, Amelink GJ, Algra A, Hofmeijer J; HAMLET Steering Committee. Surgical decompression for space-occupying cerebral infarction: outcomes at 3 years in the randomized HAMLET trial. Stroke 2013;44(09):2506-2508 
44 Jüttler E, Unterberg A, Woitzik J, et al; DESTINY II Investigators. Hemicraniectomy in older patients with extensive middle-cerebral-artery stroke. N Engl J Med 2014;370(12):1091-1100

45 Frank JI, Schumm LP, Wroblewski K, et al; HeADDFIRST Trialists. Hemicraniectomy and durotomy upon deterioration from infarction-related swelling trial: randomized pilot clinical trial. Stroke 2014;45(03):781-787

46 Zhao J, Su YY, Zhang Y, et al. Decompressive hemicraniectomy in malignant middle cerebral artery infarct: a randomized controlled trial enrolling patients up to 80 years old. Neurocrit Care 2012;17(02):161-171

47 Vahedi K, Hofmeijer J, Juettler E, et al; DECIMAL, DESTINY, and HAMLET investigators. Early decompressive surgery in malignant infarction of the middle cerebral artery: a pooled analysis of three randomised controlled trials. Lancet Neurol 2007;6(03): 215-222

48 Slezins J, Keris V, Bricis R, et al. Preliminary results of randomized controlled study on decompressive craniectomy in treatment of malignant middle cerebral artery stroke. Medicina (Kaunas) 2012;48(10):521-524

49 Kastrau F, Wolter M, Huber W, Block F. Recovery from aphasia after hemicraniectomy for infarction of the speech-dominant hemisphere. Stroke 2005;36(04):825-829

50 Solomon NA, Glick HA, Russo CJ, Lee J, Schulman KA. Patient preferences for stroke outcomes. Stroke 1994;25(09):1721-1725

51 Forsting M, Reith W, Schäbitz WR, et al. Decompressive craniectomy for cerebral infarction. An experimental study in rats. Stroke 1995;26(02):259-264

52 Doerfler A, Engelhorn T, Heiland S, Benner T, Forsting M. Perfusion- and diffusion-weighted magnetic resonance imaging for monitoring decompressive craniectomy in animals with experimental hemispheric stroke. J Neurosurg 2002;96(05): 933-940
53 Dasenbrock HH, Robertson FC, Vaitkevicius H, et al. Timing of decompressive hemicraniectomy for stroke: a nationwide inpatient sample analysis. Stroke 2017;48(03):704-711

54 Hofmeijer J, Kappelle LJ, Algra A, Amelink GJ, van Gijn J, van der Worp HB; HAMLET investigators. Surgical decompression for space-occupying cerebral infarction (the Hemicraniectomy After Middle Cerebral Artery infarction with Life-threatening Edema Trial [HAMLET]): a multicentre, open, randomised trial. Lancet Neurol 2009;8(04):326-333

55 Akins PT, Guppy KH. Sinking skin flaps, paradoxical herniation, and external brain tamponade: a review of decompressive craniectomy management. Neurocrit Care 2008;9(02):269-276

56 Sarov M, Guichard JP, Chibarro S, et al; DECIMAL investigators. Sinking skin flap syndrome and paradoxical herniation after hemicraniectomy for malignant hemispheric infarction. Stroke 2010;41(03):560-562

57 Ware JE Jr, Sherbourne CD. The MOS 36-item short-form health survey (SF-36). I. Conceptual framework and item selection. Med Care 1992;30(06):473-483

58 Indredavik B, Bakke F, Slørdahl SA, Rokseth R, Håheim LL. Stroke unit treatment improves long-term quality of life: a randomized controlled trial. Stroke 1998;29(05):895-899

59 Duncan PW, Wallace D, Lai SM, Johnson D, Embretson S, Laster LJ. The stroke impact scale version 2.0. Evaluation of reliability, validity, and sensitivity to change. Stroke 1999;30(10):2131-2140

60 Rahme R, Zuccarello M, Kleindorfer D, Adeoye OM, Ringer AJ. Decompressive hemicraniectomy for malignant middle cerebral artery territory infarction: is life worth living? J Neurosurg 2012; 117(04):749-754

61 Hofmeijer J, van der Worp HB, Kappelle LJ, Eshuis S, Algra A, Greving JP; HAMLET Steering Committee. Cost-effectiveness of surgical decompression for space-occupying hemispheric infarction. Stroke 2013;44(10):2923-2925 